\title{
A Glyoxalase-1 Knockdown Does Not Have Major Short Term Effects on Energy Expenditure and Atherosclerosis in Mice
}

\author{
Markus Wortmann, ${ }^{1}$ Maani Hakimi, ${ }^{1}$ Thomas Fleming, ${ }^{2}$ \\ Andreas S. Peters, ${ }^{1}$ Tjeerd P. Sijmonsma, ${ }^{3}$ Stephan Herzig, ${ }^{3}$ Peter P. Nawroth, ${ }^{2}$ \\ Dittmar Böckler, ${ }^{1}$ and Susanne Dihlmannn ${ }^{1}$

\begin{abstract}
${ }^{1}$ Department of Vascular and Endovascular Surgery, University of Heidelberg, 69120 Heidelberg, Germany
${ }^{2}$ Department of Medicine I and Clinical Chemistry, University of Heidelberg, 69120 Heidelberg, Germany

${ }^{3} J o i n t$ Research Division, Molecular Metabolic Control, German Cancer Research Center DKFZ,

Network Aging Research, ZMBH, and University Hospital Heidelberg, 69120 Heidelberg, Germany
\end{abstract}

Correspondence should be addressed to Markus Wortmann; markus.wortmann@med.uni-heidelberg.de

Received 2 July 2015; Accepted 27 August 2015

Academic Editor: Giovanni Annuzzi

Copyright (c) 2016 Markus Wortmann et al. This is an open access article distributed under the Creative Commons Attribution License, which permits unrestricted use, distribution, and reproduction in any medium, provided the original work is properly cited.

Objective. Glyoxalase-1 is an enzyme detoxifying methylglyoxal (MG). MG is a potent precursor of advanced glycation endproducts which are regarded to be a key player in micro- and macrovascular damage. Yet, the role of Glo1 in atherosclerosis remains unclear. In this study, the effect of Glol on mouse metabolism and atherosclerosis is evaluated. Methods. Glol knockdown mice were fed a high fat or a standard diet for 10 weeks. Body weight and composition were investigated by Echo MRI. The PhenoMaster system was used to measure the energy expenditure. To evaluate the impact of Glol on atherosclerosis, Glo1 ${ }^{\mathrm{KD}}$ mice were crossed with ApoEknockout mice and fed a high fat diet for 14 weeks. Results. Glol activity was significantly reduced in heart, liver, and kidney lysates derived from Glo1 ${ }^{\mathrm{KD}}$ mice. Yet, there was no increase in methylglyoxal-derived AGEs in all organs analyzed. The Glo1 knockdown did not affect body weight or body composition. Metabolic studies via indirect calorimetry did not show significant effects on energy expenditure. Glo1 ${ }^{\mathrm{KD}}$ mice crossed to $\mathrm{ApoE}^{-/-}$mice did not show enhanced formation of atherosclerosis. Conclusion. A Glo1 knockdown does not have major short term effects on the energy expenditure or the formation of atherosclerotic plaques.

\section{Introduction}

Diabetes is one of the major risk factors in the development of atherosclerosis. However, the link between diabetes and atherosclerosis is still poorly understood. Increasing evidence suggests that dicarbonyl stress, the abnormal accumulation of $\alpha$-oxoaldehyde metabolites during hyperglycemic metabolism, contributes to an increased risk of cardiovascular disease [1]. A recent study with more than 90.000 patients and controls identified the glyoxalase-1 (Glol) gene as a key driver of coronary artery disease [2].

Glol is a cytosolic protein that forms, together with glyoxalase 2 and glutathione, the glyoxalase system. The main function of this system is the detoxification of reactive dicarbonyls, in particular methylglyoxal (MG), which forms from spontaneous degradation of triosephosphates [3]. MG modifies arginine residues of proteins, resulting in formation of so-called advanced glycation end products (AGEs). An age-dependent decline in Glol activity has been shown to cause increased MG plasma levels and formation of AGEs [3]. Moreover, both, accumulation of $\mathrm{MG}$ and increased AGE levels, have been shown to be involved in a number of several microvascular complications of diabetes mellitus, for example, nephropathy [4] and retinopathy [5].

As recently reviewed by us [6], there is increasing evidence that the balance between production of MG and its detoxification by Glol might be involved in the formation and progression of atherosclerotic lesions. In addition, chemical inhibition of Glol has been shown to induce atherosclerosis in ApoE deficient mice [7]. In this study, Glol was further investigated in an in vivo model of Glo1 knockdown ( $\left.\mathrm{Glol}^{\mathrm{KD}}\right)$ 
mice, to determine its distinct role in metabolism under different mouse diets and progression of atherosclerosis.

\section{Materials and Methods}

2.1. Animals and Genotyping. The transgenic mouse line B6.129P2-Apoe $e^{\text {tmlUnc }}\left(\mathrm{ApoE}^{-/-}\right)$was purchased from Jackson laboratories; the transgenic Glol knockdown mice $\left(\mathrm{Glol}^{\mathrm{KD}}\right)$ were a kind gift of the lab of Michael Brownlee [8]. For the atherosclerosis experiments, $\mathrm{Glol}^{\mathrm{KD}}$ mice were bred onto the $\mathrm{ApoE}^{-/-}$mice. Male double transgenic mice $\left(\mathrm{Glol}^{\mathrm{KD}} \times\right.$ $\mathrm{ApoE}^{-/-}$) were compared to age-matched male $\mathrm{ApoE}^{-/-}$ and Glo1 ${ }^{\mathrm{KD}}$ littermates. For genotyping, genomic DNA was extracted from mouse tails and analyzed by PCR. Primers used are listed in Supplementary Table 1 (see Supplementary Table 1 in the Supplementary Material available online at http://dx.doi.org/10.1155/2016/2981639). Animals were fed a standard chow for 10 weeks. Subsequently, half the animals in each group $(n=12)$ were placed on a high fat diet for another 14 weeks to induce obesity and atherosclerosis as indicated in the figures and text. Animals receiving standard chow diet $(n=12)$ for the entire experimental period were used as controls.

2.2. Analysis of Feeding Behavior, Locomotor Activity, and Metabolic Parameters. Ten-week-old wild-type and Glo1 ${ }^{\mathrm{KD}}$ mice were fed experimental diets with $60 \%$ calories derived from fat (in the following referred to as high fat diet) and $10 \%$ calories derived from fat (in the following referred to as low fat diet) (resp., D12492 or D12450B, Research Diets, New Brunswick) for 10 weeks (= four groups with five animals each). Body weight was monitored weekly for eight weeks until the mice entered the PhenoMaster System (TSE systems $\mathrm{GmbH}$, Bad Homburg, Germany). After leaving the PhenoMaster System, an Echo-MRI analysis (Echo Medical System, Houston, TX, USA) was applied to determine the body composition of the mice. Data analysis was performed as described [9].

2.3. Tissue Preparation and Quantification of Glo1 Activity. Heart, liver, and kidneys were removed from 22-week-old $\mathrm{Glol}^{\mathrm{KD}}$ mice and wild-type littermates, both fed with a standard chow diet. Tissues were immediately flash frozen in liquid nitrogen and stored at $-80^{\circ} \mathrm{C}$. Tissue homogenization, preparation of total protein extracts, and analysis of Glo-1 activity were performed as previously described [10].

2.4. Glo1 Western Blotting. Protein extracts $(20 \mu \mathrm{g})$ were separated under denaturing conditions on precast $4 \%$ to $20 \%$ mini gels, transferred to a nitrocellulose membrane, and blocked with 5\% non-fat dry milk. Membranes were incubated overnight with rabbit-anti-Glo1 (1:1000; ab96032, Abcam, Cambridge, UK) or for one hour with mouseanti-actin (1:5000; MP Biomedicals, Eschwege, Germany). Membranes were subsequently incubated with the appropriate horseradish peroxidase conjugated secondary antibody (1:5000; Jackson ImmunoResearch Laboratories, Europe; Dianova, Hamburg, Germany). Immunoreactive proteins were visualized on X-ray films using enhanced chemiluminescence detection reagents, according to the manufacturer's instructions. Densitometric analysis (ImageJ) was used to determine relative Glol levels, normalized to actin as a loading control.

2.5. Analysis of Methylglyoxal Derivatives. Frozen excised tissues from heart, liver, and kidneys were ground to a fine powder under liquid nitrogen and resuspended in ice cold lysis buffer (60 mM Na2HPO4, $40 \mathrm{mM}$ NaH2PO4, $10 \mathrm{mM}$ $\mathrm{KCl}, 1 \mathrm{mM} \mathrm{MgSO} 4, \mathrm{pH}$ 7.0). Extracts were incubated on ice for $10 \mathrm{~min}$, homogenized with a syringe and a fine needle, and centrifuged $\left(18000 \mathrm{~g}, 4^{\circ} \mathrm{C}\right)$. Supernatants were used for analysis of methylglyoxal-derived AGEs by ELISA (OxiSelect methylglyoxal (MG) competitive ELISA Kit, Cell Biolabs Inc., San Diego, USA) according to the recommendations of the manufacturer. Briefly, undiluted cell lysates were loaded to MG-conjugate coated plates and detected by AntiMG antibody, secondary antibody, and substrate solution. Absorbance was read in a microplate reader at $450 \mathrm{~nm}$ as primary wave length.

2.6. Blood Glucose and Plasma Lipid Quantification. Blood was sampled from the heart in EDTA-coated microvettes to prevent coagulation. Blood glucose was determined immediately with fresh blood by using an Accu Check Aviva blood glucose meter (Roche, Mannheim, Germany). After centrifugation, plasma was removed and stored at $-80^{\circ} \mathrm{C}$ until further analysis. Triglycerides and cholesterol were determined as previously described [11] using standard analysis methods at the Central Diagnostics Laboratory of the University Hospital Heidelberg.

2.7. Characterization and Quantification of Atherosclerotic Lesions. En face preparations of the aortic arch were performed following a standard protocol. Briefly, mice were lethally anesthetized using inhalation anesthesia (isoflurane) and the complete aortas were removed together with the heart. Using a stereo microscope, aortas were dissected from any perivascular fat and connective tissue and subsequently sliced longitudinally. Aortas were fixed in 80\% 2-propanol and stained with oil red O (Sigma-Aldrich, Steinheim, Germany; $0.5 \%$ in 2-propanol) for $10 \mathrm{~min}$. After differentiating in $80 \%$ 2-propanol for 2-5 min, en face pictures were taken for digital analysis. ImageJ was used to determine red stained areas in relation to the total area of the aortic arch or the complete aorta, respectively. For analysis of the aortic sinus, the upper part of the heart was formalin-fixed and embedded in paraffin. Transversal sections of the aortic sinus were prepared and MOVAT staining was performed (detailed staining protocols are available on request). Quantification of atherosclerosis was performed by analysis of MOVATstained cross sections of the aortic sinus. ImageJ was used to determine the remaining lumen in relation to the total area of the aortic sinus.

2.8. Immunohistochemical Detection of Glo1. Serial $2 \mu \mathrm{m}$ transversal sections were prepared from formalin-fixed and paraffin-embedded aortic sinus specimens. After 


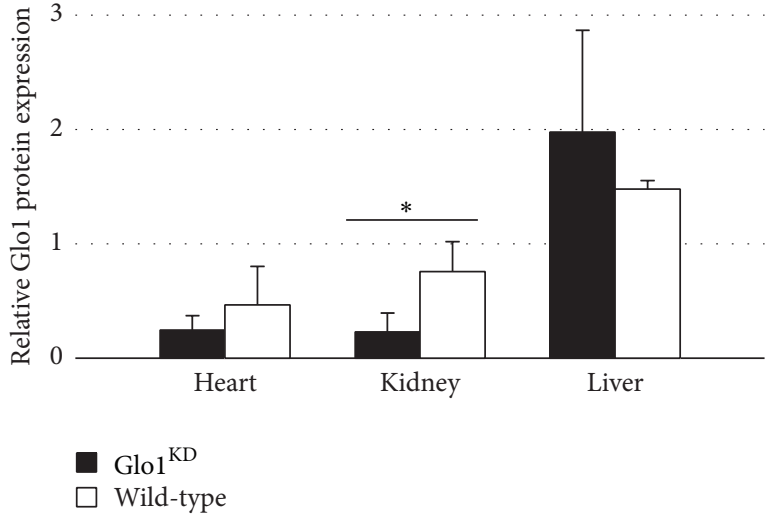

(a)

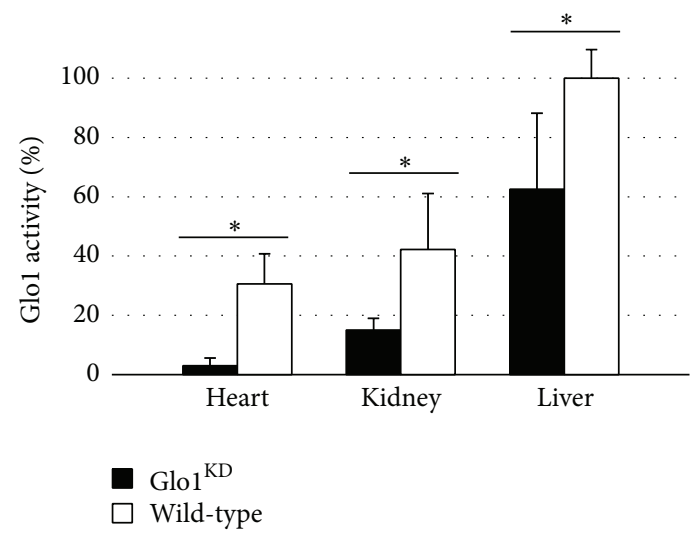

(b)

0.8

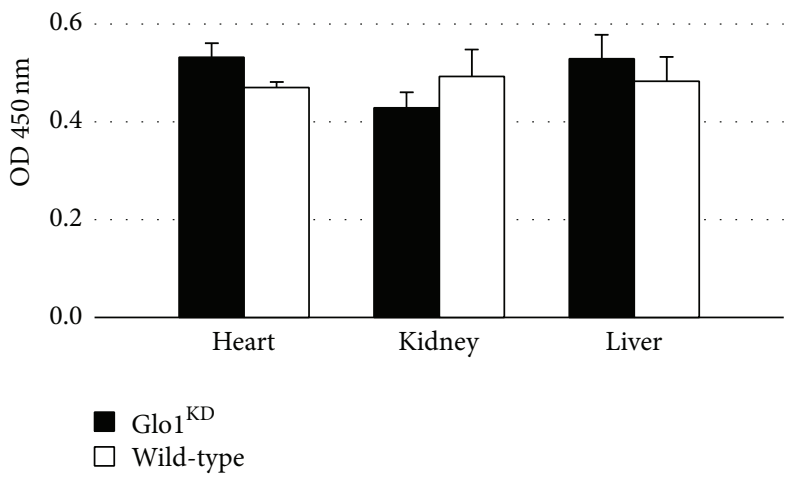

(c)

FIGURE 1: (a) Glol protein levels measured via Western blot were significantly reduced in the kidney $(p=0.0096)$, whereas protein levels in the heart $(p=0.27)$ and the liver $(p=0.34)$ did not differ significantly. (b) Glol activity was significantly reduced in the heart ( $p=0.001)$, kidney $(p=0.03)$, and liver $(p=0.03)$ of $\mathrm{Glol}^{\mathrm{KD}}$ mice in comparison to wild-type animals $(n=4)$. Glol activity in the liver of wild-type mice was arbitrarily set to $100 \%$. (c) The levels of methylglyoxal-derived AGEs were measured via an ELISA. There was no significant impact of the Glo1 knockdown on the methylglyoxal-derived AGEs in extracts of heart, kidney, and liver $(p \geq 0.05)$.

deparaffinization and rehydration, sections were pretreated in target retrieval solution $\mathrm{pH}$ 6.0 (DAKO, Glostrup, Denmark) in a steamer for $30 \mathrm{~min}$. After $5 \mathrm{~min}$ washing in TBS buffer (20 mM Tris, $137 \mathrm{mM} \mathrm{NaCl}, \mathrm{pH} 7.6$ ) sections were incubated overnight with anti-Glo1 (1:300; ab96032, Abcam, Cambridge, UK). The DAKO real detection system peroxidase/AEC Rabbit/Mouse (DAKO, Glostrup, Denmark), containing biotinylated secondary antibody, streptavidin$\mathrm{HRP}$, and AEC/H2O2 substrate, was applied for further treatment, following the manufacturer's recommendation. Counterstaining was performed for $30 \mathrm{sec}$ with Mayer's hemalum solution (Merck Darmstadt, Germany).

2.9. Statistical Analysis. Statistical analysis was performed using SigmaStat 2.0 software (Jandel GmbH, Erkrath, Germany) or SPSS Statistics version 22 (IBM, Armonk, USA). Experimental values are expressed as mean \pm SD unless otherwise stated. Statistically significant differences between groups were calculated by Student's $t$-test or two-way ANOVA. Values of $p<0.05$ were considered significant.

\section{Results}

3.1. The Glo1 Knockdown Results in a Significantly Reduced Protein Level in the Kidney and a Significantly Reduced Glo1 Activity in Heart, Kidney, and Liver. Adult Glo1 ${ }^{\mathrm{KD}}$ mice were sacrificed and Glol protein levels as well as Glol activity were measured in the heart, liver, and kidney. Glol protein levels were measured via Western blotting in relation to actin. Glo1 protein levels were significantly reduced in kidney $\left(\mathrm{Glol}^{\mathrm{KD}}\right.$ versus wild-type: $0.231 \pm 0.165$ versus $0.757 \pm 0.264, p=$ $0.0096)$, whereas protein levels in heart ( $\mathrm{Glol}^{\mathrm{KD}}$ versus wildtype: $0.246 \pm 0.128$ versus $0.467 \pm 0.335, p=0.27$ ) and liver (Glo1 $^{\mathrm{KD}}$ versus wild-type: $1.977 \pm 0.892$ versus $1.480 \pm 0.072$, $p=0.34$ ) were not significantly different (Figure 1(a)).

Glo1 activity was reduced in liver lysates by $38 \%(62.5 \% \pm$ $25.7 \%$ versus $100 \% \pm 9.7 \%, p=0.03$ ), in kidney lysates by $64 \%(15.1 \pm 3.9 \%$ versus $42.2 \pm 18.8 \%, p=0.03)$, and in heart lysates by $90 \%(3 \pm 2.6 \%$ versus $30.6 \pm 10.1 \%, p=0.001)$ in comparison to wild-type mice. Glol activity in the liver of wild-type mice was arbitrarily set to 100\% (Figure 1(b)). 
TABLE 1: The body composition was measured via Echo-MRI. There was no significant difference in the total body weight nor in the relative fat or lean mass $(p \geq 0.05)$.

\begin{tabular}{|c|c|c|c|c|c|}
\hline Genotype & Diet & Weight (g) & Fat $(\%)$ & Lean (\%) & Rest (\%) \\
\hline $\mathrm{Glo1}^{\mathrm{KD}}$ & High fat diet & $40.8 \pm 3.32$ & $45.1 \pm 3.0$ & $49.5 \pm 2.8$ & $5.4 \pm 0.5$ \\
\hline Wild-type & High fat diet & $42.4 \pm 1.96$ & $43.2 \pm 0.9$ & $50.9 \pm 0.9$ & $5.9 \pm 0.8$ \\
\hline $\mathrm{Glo1}^{\mathrm{KD}}$ & Low fat diet & $27.9 \pm 1.57$ & $24.3 \pm 4.6$ & $67.4 \pm 2.9$ & $8.3 \pm 1.8$ \\
\hline Wild-type & Low fat diet & $28.7 \pm 2.44$ & $22.2 \pm 3.7$ & $69.6 \pm 3.6$ & $8.2 \pm 1.3$ \\
\hline
\end{tabular}

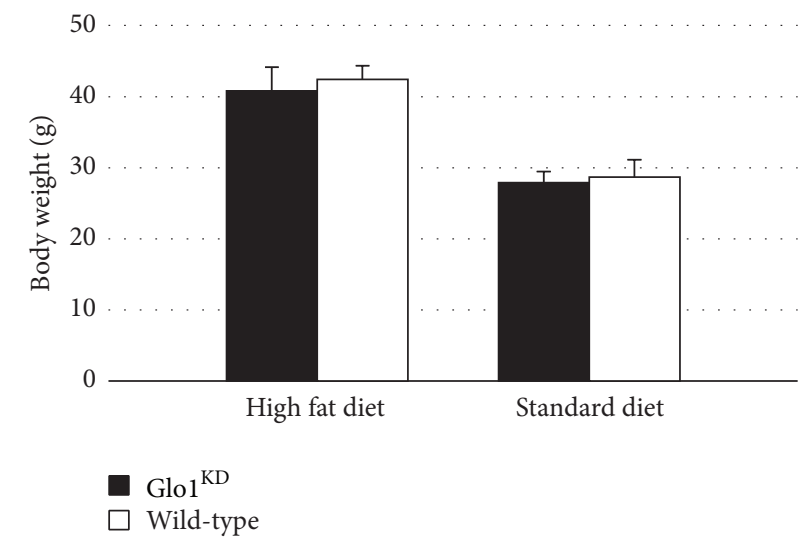

(a)

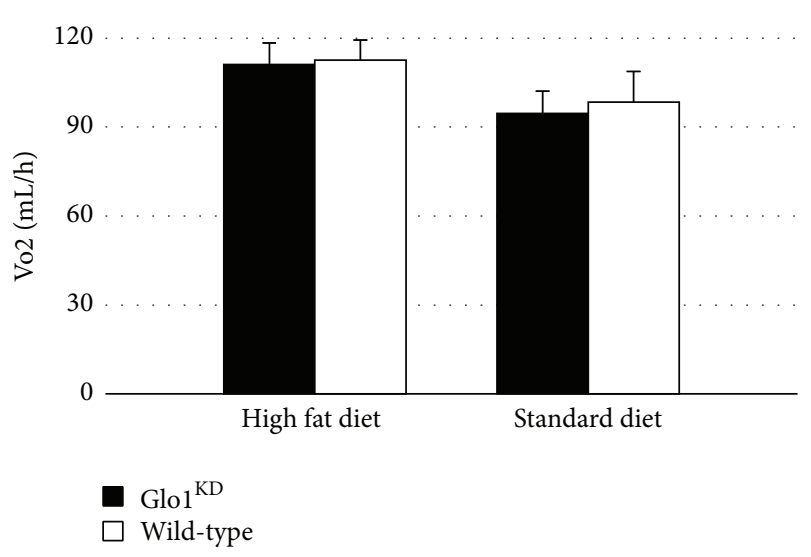

(b)

FIGURE 2: (a) The mean body weight did not differ between $\mathrm{Glol}^{\mathrm{KD}}$ mice and wild-type mice, neither on high fat diet $(p=0.654)$ nor on standard diet. (b) The mean oxygen consumption over $24 \mathrm{~h}$ was measured using the PhenoMaster system. The genotype did not have a significant influence $(p \geq 0.05)$.

3.2. Levels of Methylglyoxal-Derived AGEs Were Not Elevated in Glol $^{K D}$ Mice. In order to measure the levels of methylglyoxal-derived AGEs, an ELISA measuring methylglyoxal-hydroimidazolone was performed. Although Glol activity was significantly reduced in all organs analyzed, the levels of methylglyoxal-derived AGEs were not affected in liver (high fat diet: $2.8 \pm 0.36 \mu \mathrm{g} / \mathrm{mL}$ versus $3.3 \pm 0.42 \mu \mathrm{g} / \mathrm{mL}$, $p=0.077$; standard chow: $2.1 \pm 0.33 \mu \mathrm{g} / \mathrm{mL}$ versus $3.1 \pm$ $0.09 \mu \mathrm{g} / \mathrm{mL}, p=0.13$ ), kidney (high fat diet: $2.9 \pm 0.33 \mu \mathrm{g} / \mathrm{mL}$ versus $2.2 \pm 0.3 \mu \mathrm{g} / \mathrm{mL}, p=0.061$; standard chow: $2.94 \pm$ $0.29 \mu \mathrm{g} / \mathrm{mL}$ versus $4.3 \pm 0.65 \mu \mathrm{g} / \mathrm{mL}, p=0.076)$, and heart (high fat diet: $5.5 \pm 0.91 \mu \mathrm{g} / \mathrm{mL}$ versus $7.5 \pm 1.1 \mu \mathrm{g} / \mathrm{mL}, p=$ 0.36 ; standard chow: $5.8 \pm 0.97 \mu \mathrm{g} / \mathrm{mL}$ versus $8.0 \pm 1.25 \mu \mathrm{g} / \mathrm{mL}$, $p=0.091$ ) lysates (Figure 1(c)).

3.3. Glo1 ${ }^{K D}$ Mice Do Not Show Major Alterations in Feeding Behavior, Locomotor Activity, and Different Metabolic Parameters. Glo1 ${ }^{\mathrm{KD}}$ mice as well as $\mathrm{C} 57 \mathrm{Bl} / 6$ mice, which served as control animals, were fed a standard chow or alternatively a high fat diet for 10 weeks (5 mice per groups). During this time the body weight was measured weekly. The weight gain of the Glo1 ${ }^{\mathrm{KD}}$ mice, in relation to the weight at the beginning of the experiment, did not differ from the weight gain of wildtype mice after 10 weeks ( $\mathrm{Glol}^{\mathrm{KD}}$ versus wild-type, standard chow: $154 \pm 7 \%$ versus $143 \pm 5 \%, p=0.241$; Glol $^{\mathrm{KD}}$ versus wild-type, high fat diet: $213 \pm 11 \%$ versus $220 \pm 10 \%, p=$ $0.654)$. The same was true for the absolute body weight after 10 weeks (Glo1 ${ }^{\mathrm{KD}}$ versus wild-type, standard chow: $40.8 \pm 3.32 \mathrm{~g}$ versus $42.4 \pm 1.96 \mathrm{~g}, p=0.379$; $\mathrm{Glo1}{ }^{\mathrm{KD}}$ versus wild-type, high fat diet: $27.9 \pm 1.57 \mathrm{~g}$ versus $28.7 \pm 2.44 \mathrm{~g}, p=0.547$ ) (Figure 2(a)).

Ten weeks after the beginning of the experiment, feeding behavior, locomotor activity, and the energy expenditure were measured using the PhenoMaster system. The knockdown of Glol did not cause any major metabolic alterations, regarding neither oxygen consumption (Figure 2(b)) nor food intake or activity (Supplemental Figure S1).

After eleven weeks, nuclear magnetic resonance (NMR) relaxometry was performed via the Echo-MRI system to analyze the body composition. There was no significant difference in the total bodyweight or in the relative fat or lean weight $(p \geq 0.05)$ (Table 1$)$.

3.4. Double Transgenic Glo1 ${ }^{K D} \times A p o E^{-/-}$Mice Do Not Suffer from Enhanced Atherosclerosis. The double transgenic $\left(\mathrm{Glol}^{\mathrm{KD}} \times \mathrm{ApoE}^{-/-}\right)$mice received a high fat diet for 10 weeks in order to induce formation of atherosclerotic plaques. The Glo1 knockdown did not cause alterations in the triglyceride, cholesterol, or blood sugar levels (Figures 3(a), 3(b), and $3(\mathrm{c})$ ). Also the gain of weight was not affected by the Glo1 knockdown (data not shown). With respect to atherosclerosis, the knockdown of Glol did not enhance the formation of atherosclerotic plaques in the aortic arch analyzed via en face preparations (Figure 4(a)). Similar findings were also obtained for en face preparations of the whole aorta (Supplemental Figure 2) and the plaque area in histological 


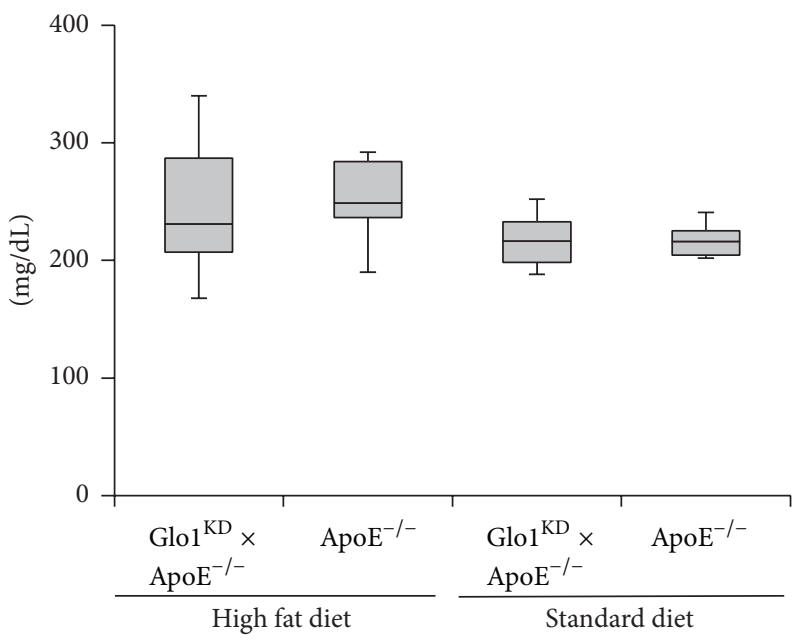

(a)

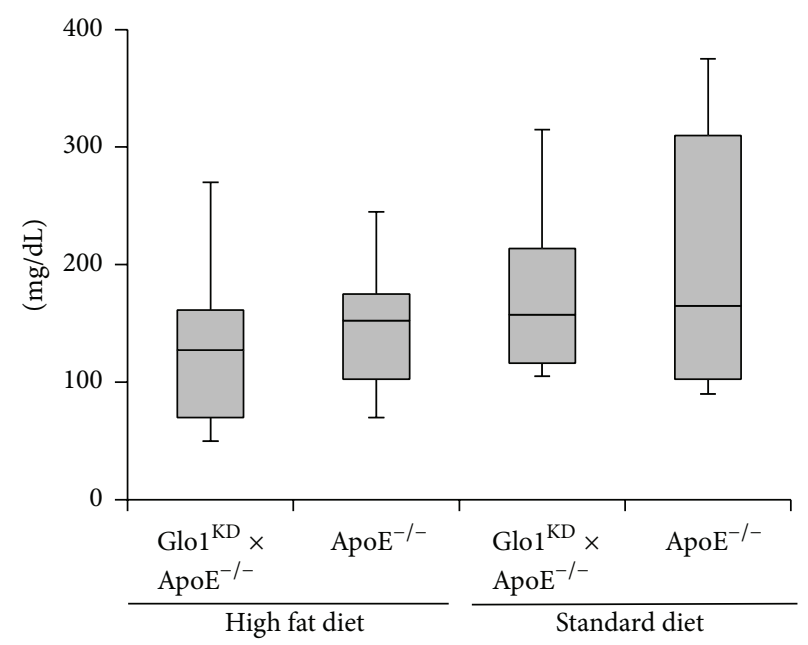

(b)

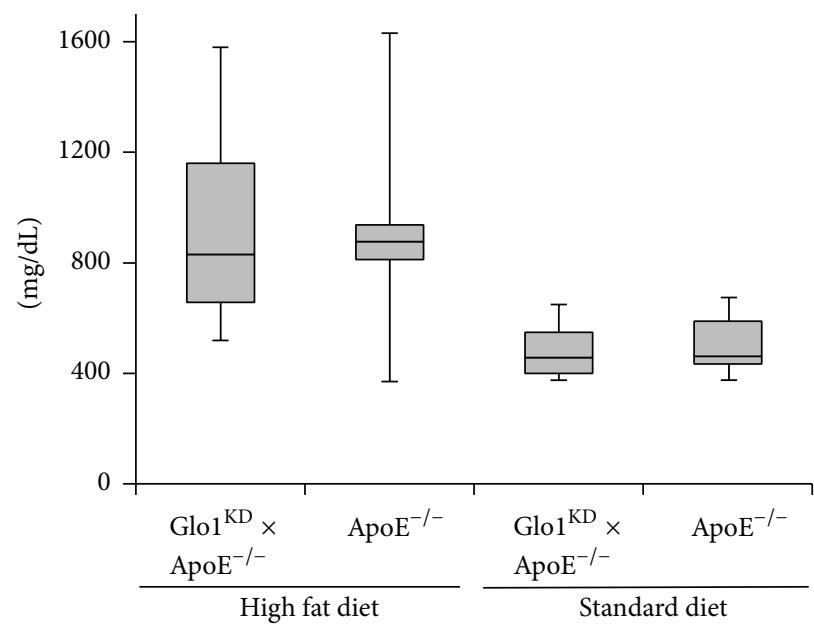

(c)

Figure 3: Blood glucose (a), triglycerides (b), and cholesterol (c) were measured in $\mathrm{Glol}^{\mathrm{KD}} \times \mathrm{ApoE}^{-/-}$and ApoE ${ }^{-/-}$mice, both on a high fat and on a standard diet. The Glol activity did not have an impact on all parameters measured.

sections of the aortic sinus (Figure 4(b)). Immunohistochemical analysis of the aortic sinus for detection of Glo1 expression revealed a similar distribution of Glol-positive areas in all phenotypes, irrespective of the diet. Glo1 was mainly detected within the plaques and the myocardium, whereas little or no Glo1 expression was detected within the aortic wall (Figure 4(c)).

\section{Discussion}

A reduced capacity of Glo1 to detoxify methylglyoxal is associated with endothelial dysfunction, nephropathy, and neuropathy, all of which represent important features of microvascular complications associated with diabetes mellitus.

However, the function of Glo1 in macrovascular disease, particularly atherosclerosis, still remains uncertain [6]. While large epidemiologic studies have not been able to detect effects of different SNPs of the Glo1 gene on formation of atherosclerosis [12, 13], Hanssen et al. reported higher levels of advanced glycation end products in human carotid atherosclerotic plaques associated with a higher risk of plaque rupture [14].

Even less is known about the role of Glol in metabolism, particularly in hyperlipidaemia being one of the main risk factors for atherosclerosis.

The aim of this study was to specify the role of Glo1 in mouse metabolism under the challenge of different diets (high fat diet versus standard chow) and its role in the formation of atherosclerotic plaques. Considering its role in detoxifying methylglyoxal, a potent precursor of MG-derived AGEs, we expected an enhanced formation of atherosclerotic plaques in $\mathrm{Glol}^{\mathrm{KD}}$ mice. Furthermore, significant changes to the mouse metabolism were assumed.

In this study, the Glol knockdown caused a significant reduction of Glol protein levels in tissue extracts derived from the kidney, whereas protein levels in heart and liver extracts were not affected. Nevertheless, Glol activity was 


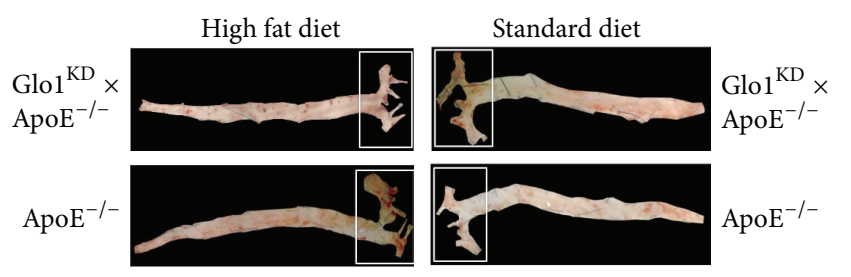

Oil red O staining of aortic arch

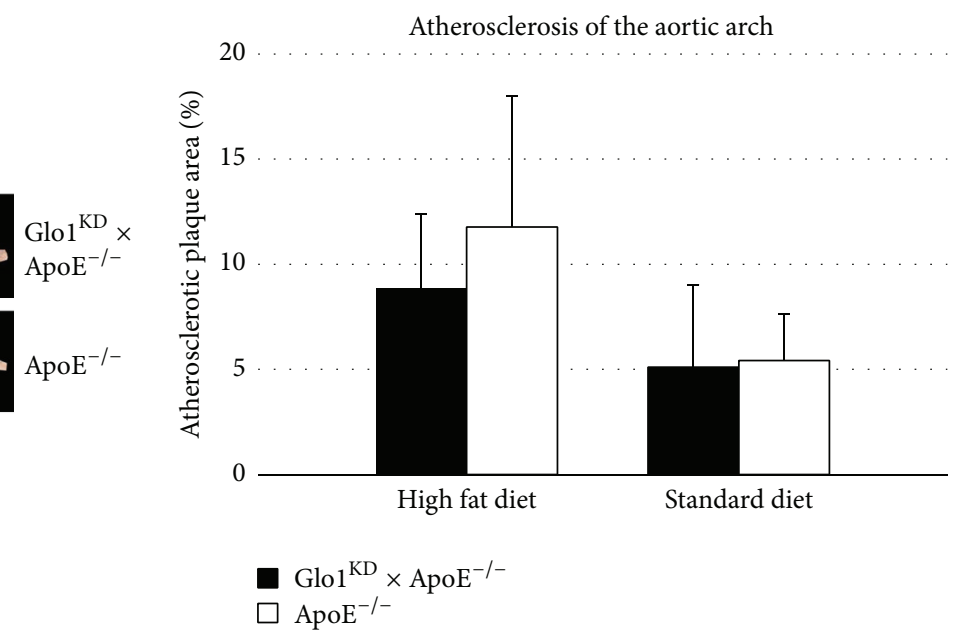

(a)

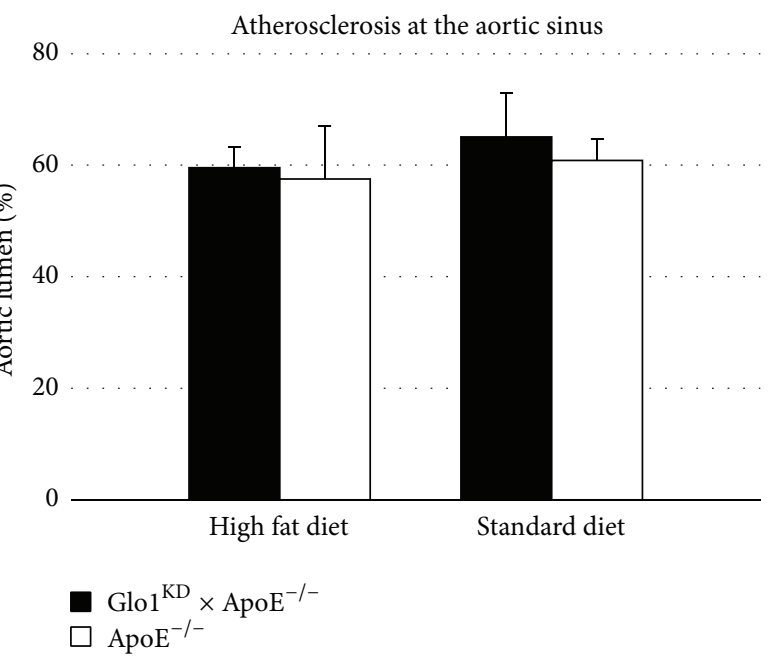

(b)

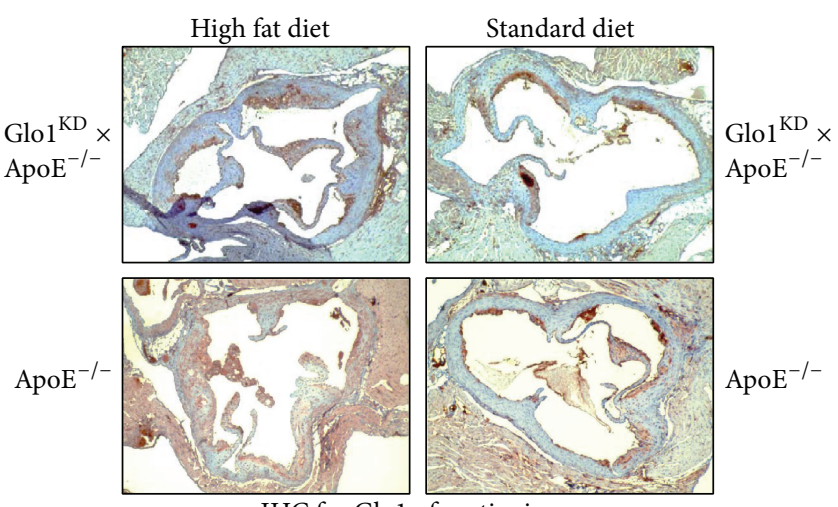

IHC for Glo1 of aortic sinus

(c)

FigurE 4: (a) En face preparations were performed in order to quantify the amount of atherosclerotic plaques. Bars refer to the mean of oil red $\mathrm{O}$ positive areas, analyzed in relation to the total area of the aortic arch \pm standard deviation. The knockdown of Glol did not show any significant effect on the formation of atherosclerotic plaques $(p \geq 0.05)$. (b) Transversal sections of the aortic sinus were stained via a MOVAT staining in order to analyze the atherosclerotic plaque formation. Bars represent the mean of the aortic lumina (as determined by ImageJ analysis, lumen area in relation to the total area surrounded by the outer media). The Glol knockdown did not have a significant impact of the stenosis caused by atherosclerotic plaques $(p \geq 0.05)$. (c) Immunohistochemical detection of Glo1 performed with transversal sections of the aortic sinus. Glol was mainly detected in the atherosclerotic plaques. 
significantly diminished in all organ extracts named by $38 \%-$ $90 \%$ which agrees well with previously published data for the same strain of $\mathrm{Glol}^{\mathrm{KD}}$ mice [8].

Since the Glol activity assays have been performed under in vitro conditions, supplemented with reduced glutathione, we cannot conclude from our data whether the reduced Glo1 activities are reflected under in vivo conditions as well, and whether they resulted in increased accumulation of AGEs.

The levels of MG-derived AGEs were determined via a MG-H1 ELISA in the lysates of kidney, liver, and heart. Surprisingly, MG-derived AGE levels were unaffected in the knockdown mice. This was in line with data from Geoffrion et al., who did not detect increased AGE levels, represented by glycated aortal collagen, in the same mouse strain [15]. It should be noted however, that this study is slightly limited by the use of imprecise methods for determination of MGderived AGEs since the ELISA technique used is hardly reproducible and not quantitative. Future analysis, using analytical mass spectrometry, will help to better define and quantify the accumulation of MG-derived AGEs.

$\mathrm{Glol}^{\mathrm{KD}}$ mice did not show any major metabolic aberrations. Neither in regard to surrogate parameters, such as oxygen consumption or resting metabolic rate (analyzed in metabolic cages), nor in the body weight or body composition, which were measured by Echo-MRI, a significant impact of the Glo1 knockdown was detectable. Even challenging the metabolism with a high fat diet, bearing in mind that more methylglyoxal may be accumulating during hyperlipidaemic metabolism, did not reveal any impact of Glol on mouse metabolism.

In order to study the role of Glol in the progress of atherosclerosis, double transgenic $\mathrm{Glol}^{\mathrm{KD}} \times \mathrm{ApoE}^{-/-}$mice were created. These mice, compared to age-matched $\mathrm{ApoE}^{-/-}$ mice, did not show any significant difference in weight, cholesterol, or triglyceride levels after being fed a high fat diet or standard chow for 10 weeks.

The $\mathrm{ApoE}^{-/-}$mouse strain used in this experiment mainly suffers from atherosclerotic lesions in the aortic sinus as well as in the proximal aorta [16, 17]. Increased AGE accumulation caused by reduction of Glol activity should thus result in larger or faster plaque formation. In contrast, the Glo1 knockdown in double transgenic mice did not have any impact on atherosclerosis of the upper aorta. Atherosclerotic lesions in the infrarenal aorta were scarcely observed in all mouse strains, and additional analysis of atherosclerotic plaques of the complete aorta did not show an impact of the Glol knockdown, either.

While in this study the Glol activity was reduced via a knockdown model, Hanssen et al. have crossed Glol overexpressing mice with $\mathrm{ApoE}^{-/-}$mice in order to evaluate whether a higher Glol activity might reduce the formation of atherosclerotic plaques. Consistent with our results, there was no protective effect of the Glol overexpression, neither under regular conditions nor under diabetic conditions [18].

During the preparation of this paper, Geoffrion et al. have published a study in which both Glol knockdown mice and Glol overexpressing mice were evaluated with regard to the formation of atherosclerotic lesions [15]. In line with our results, neither an enhanced nor a reduced Glol activity did have any effects on atherosclerosis, even after streptozotocin induced diabetes.

Considering the lack of MG-derived AGE accumulation in the $\mathrm{Glol}^{\mathrm{KD}}$ mice of our study, the reduction in Glo1 activity may not suffice to cause pathological consequences in macrovascular disease. Alternatively, the reduced detoxification rate by Glol might be replaced by other enzymatic reactions. For example, aldo-keto reductase has recently been shown to significantly contribute to MG-detoxification in various cell types, particularly when glutathione is limited in tissues and oxidative stress is increased $[19,20]$. It will be interesting to see whether this enzyme or other mechanisms can overcome effects of reduced Glol activity in the context of atherosclerosis. Future studies using Glol knockout mice with a complete reduction of the Glol activity will be needed in order to finally work out the role of Glol in atherosclerosis and metabolism.

In summary, the downregulation of Glol activity via a Glol knockdown did not have any measurable effects on the weight, body composition, or metabolism. Furthermore, no significant effect on the formation of atherosclerotic lesions in the aorta in double transgenic $\mathrm{Glol}^{\mathrm{KD}} \times \mathrm{ApoE}^{-/-}$mice could be detected.

\section{Conflict of Interests}

The authors declare that there is no conflict of interests.

\section{Authors' Contribution}

Markus Wortmann and Maani Hakimi contributed equally to this work.

\section{Acknowledgments}

The authors kindly thank M. Brownlee for providing the Glo1 knockdown mice. They thank Anja Spieler and Axel Erhardt for excellent technical assistance.

\section{References}

[1] N. Rabbani and P. J. Thornalley, "Dicarbonyl stress in cell and tissue dysfunction contributing to ageing and disease," Biochemical and Biophysical Research Communications, vol. 458, no. 2, pp. 221-226, 2015.

[2] V.-P. Mäkinen, M. Civelek, Q. Meng et al., "Integrative genomics reveals novel molecular pathways and gene networks for coronary artery disease," PLoS Genetics, vol. 10, no. 7, Article ID e1004502, 2014.

[3] M. Xue, N. Rabbani, and P. J. Thornalley, "Glyoxalase in ageing," Seminars in Cell and Developmental Biology, vol. 22, no. 3, pp. 293-301, 2011.

[4] O. Brouwers, P. M. G. Niessen, T. Miyata et al., "Glyoxalase-1 overexpression reduces endothelial dysfunction and attenuates early renal impairment in a rat model of diabetes," Diabetologia, vol. 57, no. 1, pp. 224-235, 2014.

[5] A. K. Berner, O. Brouwers, R. Pringle et al., "Protection against methylglyoxal-derived AGEs by regulation of glyoxalase 
1 prevents retinal neuroglial and vasodegenerative pathology," Diabetologia, vol. 55, no. 3, pp. 845-854, 2012.

[6] M. Wortmann, A. S. Peters, M. Hakimi, D. Böckler, and S. Dihlmann, "Glyoxalase I (Glol) and its metabolites in vascular disease," Biochemical Society Transactions, vol. 42, no. 2, pp. 528-533, 2014.

[7] C. Tikellis, R. J. Pickering, D. Tsorotes et al., "Dicarbonyl stress in the absence of hyperglycemia increases endothelial inflammation and atherogenesis similar to that observed in diabetes," Diabetes, vol. 63, no. 11, pp. 3915-3925, 2014.

[8] A. El-Osta, D. Brasacchio, D. Yao et al., "Transient high glucose causes persistent epigenetic changes and altered gene expression during subsequent normoglycemia," The Journal of Experimental Medicine, vol. 205, no. 10, pp. 2409-2417, 2008.

[9] M. H. Tschöp, J. R. Speakman, J. R. S. Arch et al., "A guide to analysis of mouse energy metabolism," Nature Methods, vol. 9, no. 1, pp. 57-63, 2012.

[10] T. H. Fleming, T.-M. Theilen, J. Masania et al., "Agingdependent reduction in glyoxalase 1 delays wound healing," Gerontology, vol. 59, no. 5, pp. 427-437, 2013.

[11] M. von Eynatten, J. G. Schneider, P. M. Humpert et al., "Decreased plasma lipoprotein lipase in hypoadiponectinemia: an association independent of systemic inflammation and insulin resistance," Diabetes Care, vol. 27, no. 12, pp. 2925-2929, 2004.

[12] R. M. A. Henry, P. J. Kostense, A. M. W. Spijkerman et al., "Arterial stiffness increases with deteriorating glucose tolerance status: the Hoorn study," Circulation, vol. 107, no. 16, pp. 20892095, 2003.

[13] K. H. J. Gaens, C. J. H. van Der Kallen, M. M. J. van Greevenbroek, E. J. Feskens, C. D. A. Stehouwer, and C. G. Schalkwijk, "Receptor for advanced glycation end product polymorphisms and type 2 diabetes: the CODAM study," Annals of the New York Academy of Sciences, vol. 1126, pp. 162-165, 2008.

[14] N. M. J. Hanssen, K. Wouters, M. S. Huijberts et al., "Higher levels of advanced glycation endproducts in human carotid atherosclerotic plaques are associated with a rupture-prone phenotype," European Heart Journal, vol. 35, no. 17, pp. 11371146, 2014.

[15] M. Geoffrion, X. Du, Z. Irshad et al., "Differential effects of glyoxalase 1 overexpression on diabetic atherosclerosis and renal dysfunction in streptozotocin-treated, apolipoprotein Edeficient mice," Physiological Reports, vol. 2, no. 6, Article ID e12043, 2014.

[16] C. J. Hartley, A. K. Reddy, S. Madala et al., "Hemodynamic changes in apolipoprotein E-knockout mice," The American Journal of Physiology-Heart and Circulatory Physiology, vol. 279, no. 5, pp. H2326-H2334, 2000.

[17] Y. Guo, C. Zhang, X. Du, U. Nair, and T.-J. Yoo, "Morphological and functional alterations of the cochlea in apolipoprotein $\mathrm{E}$ gene deficient mice," Hearing Research, vol. 208, no. 1-2, pp. 5467, 2005.

[18] N. M. J. Hanssen, O. Brouwers, M. J. Gijbels et al., "Glyoxalase 1 overexpression does not affect atherosclerotic lesion size and severity in $\mathrm{ApoE}^{-/-}$mice with or without diabetes," Cardiovascular Research, vol. 104, no. 1, pp. 160-170, 2014.

[19] R. C. Lyon, D. Li, G. McGarvie, and E. M. Ellis, "Aldoketo reductases mediate constitutive and inducible protection against aldehyde toxicity in human neuroblastoma SH-SY5Y cells," Neurochemistry International, vol. 62, no. 1, pp. 113-121, 2013.
[20] D. L. V. Jagt and L. A. Hunsaker, "Methylglyoxal metabolism and diabetic complications: roles of aldose reductase, glyoxalase-I, betaine aldehyde dehydrogenase and 2-oxoaldehyde dehydrogenase," Chemico-Biological Interactions, vol. 143-144, pp. 341351, 2003. 


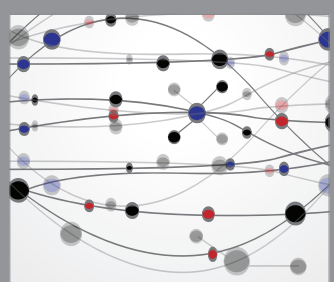

The Scientific World Journal
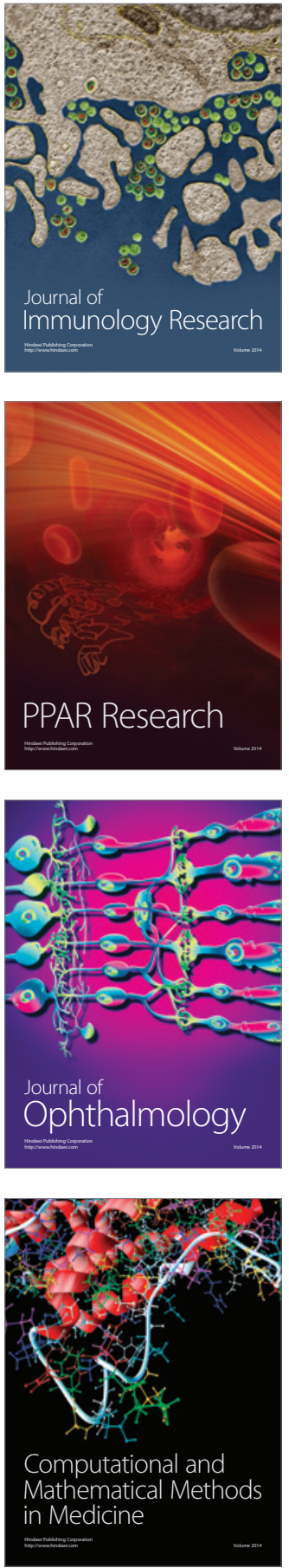

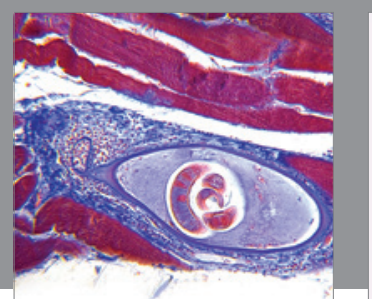

Gastroenterology Research and Practice

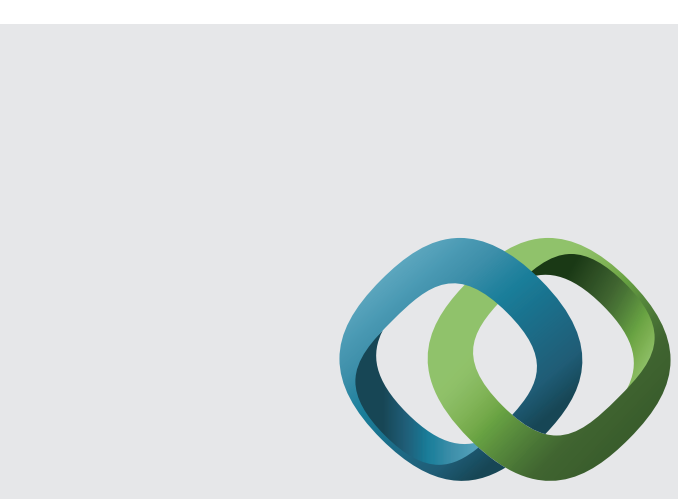

\section{Hindawi}

Submit your manuscripts at

http://www.hindawi.com
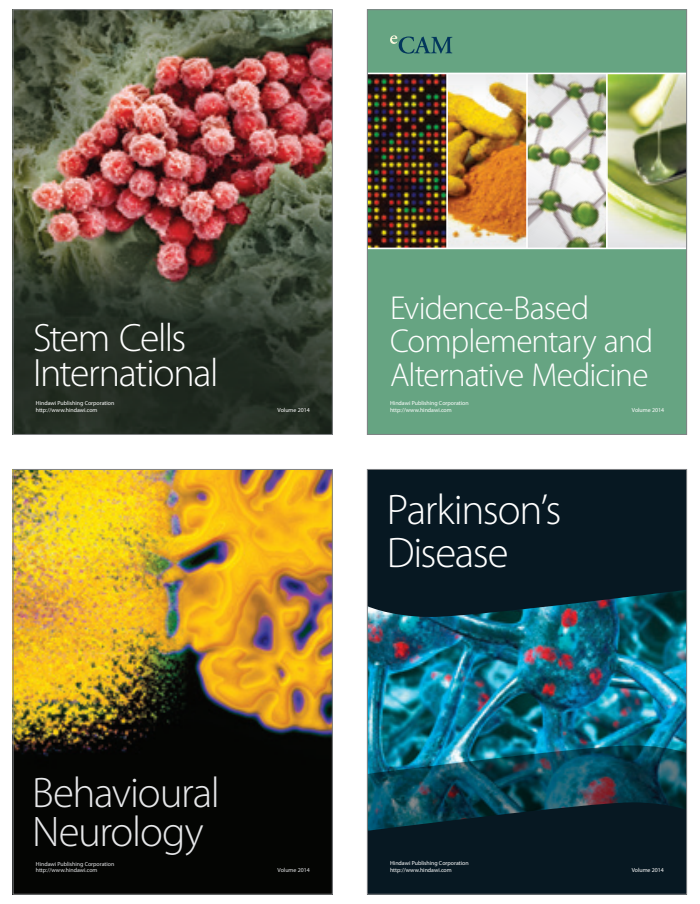
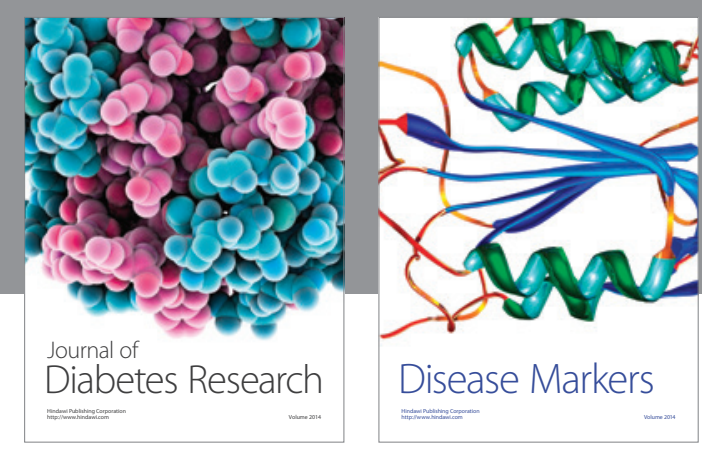

Disease Markers
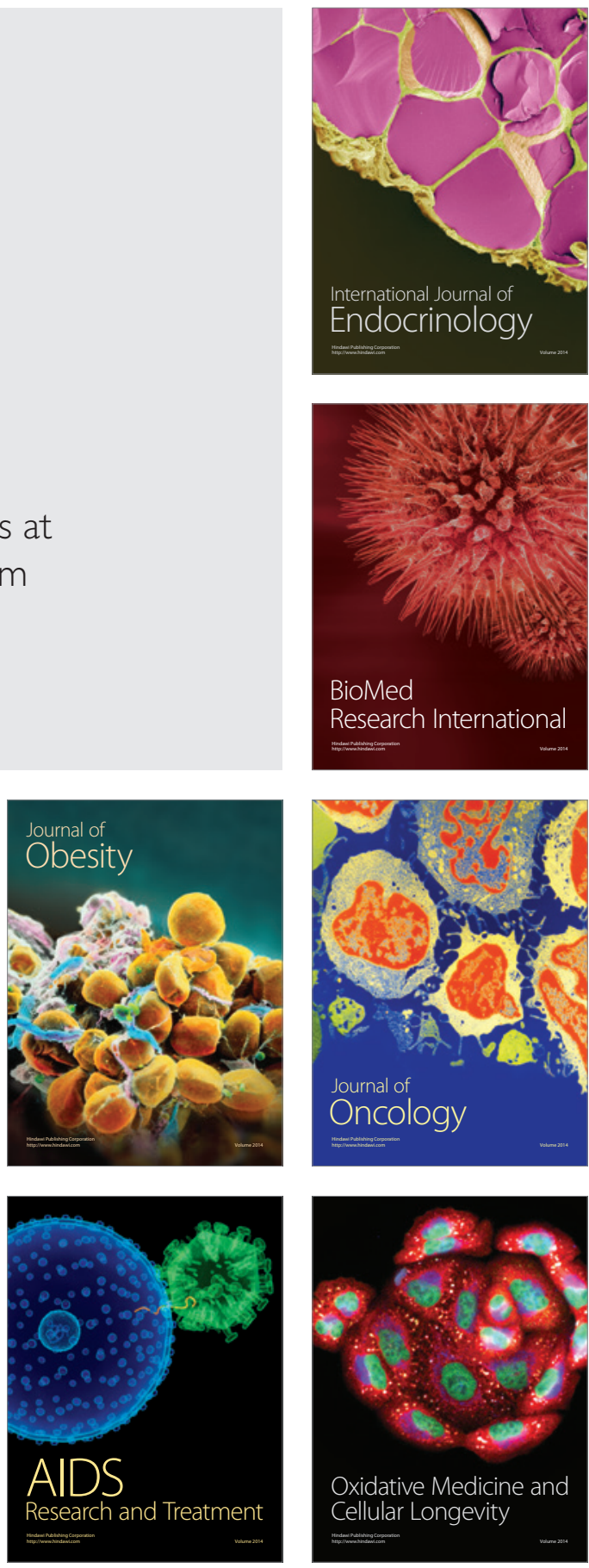